\title{
Research on Transfer Function of Electric Actuator
}

\author{
Qingsi Zhang ${ }^{1}$, Fuyun $\mathrm{Li}^{1}$, Zhitao $\mathrm{Wu}^{1}$ \\ ${ }^{1}$ School of electronic and information engineering, University of Science and Technology Liaoning, \\ Anshan 114051
}

Keywords: Actuator, Transfer function, Torque, Differential equation.

Abstract. The innovation to obtain the transfer function of the electric actuator is introduced. By this innovation, analyzing each part of the electric actuator, open-loop transfer function is obtained with voltage of power supply as the input and the feedback voltage as the output. With the solution of differential equation, a simple, fast method for obtaining the parameters of transfer function of actuator is proposed to get these parameters. The method is very quick, simple and it is proved by the experiment.

\section{Introduction}

Electric actuator is one of the automation system components, which is more and more widely applied in all walks of life. Its role in automation system is equal to man's limbs. It receives the control signal conditioner, changing the operating variables, to the normal execution of the production process according to predetermined requirements. The actuator is made up of actuator and regulating mechanism[1]. The actuator is a device that refers to the regulator control signal to generate thrust or displacement, while the regulator is a device that is based on the output signal of the actuator to change the amount of energy or material. The most common is the regulating valve. Here only study the transfer function of the actuator.

\section{Determine the form of transfer function}

\section{The basic structure of the actuator}

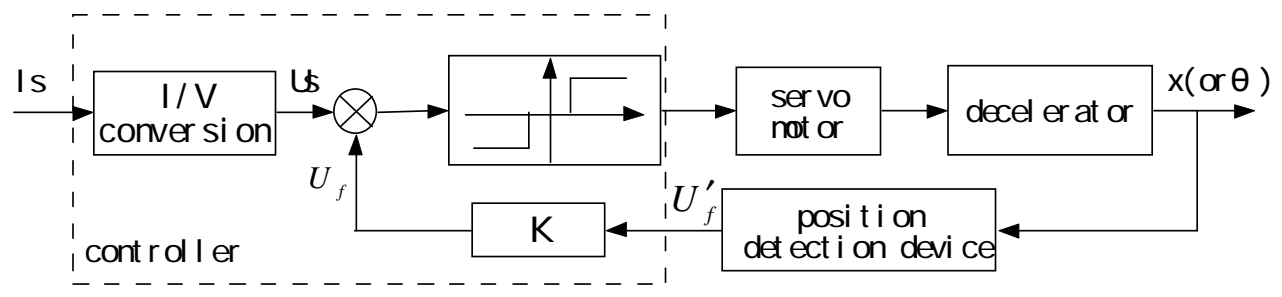

Fig1 Principle of the electric actuator

Electric actuators are shown in Figure 1, mainly including the controller (the part inside the dashed line in figure 1), servo motor, position detection device and decelerator four parts. The DC4 20mA current signal (Is) is converted to a voltage signal Us by a link(I/V conversion); The displacement signals $X$ ( or angular signal $\theta$ ) that decelerator output form feedback $\operatorname{signal}\left(U_{f}^{\prime}\right)$ after the detection of the position detecting device; The feedback signal $\left(U_{f}^{\prime}\right)$ multiplied by $\mathrm{K}$, become the position feedback $\operatorname{signal}\left(U_{f}\right)$. Us and $\mathrm{U}_{\mathrm{f}}$, two signals are through a relay - type nonlinear link to control the motor running and the motor drives the retarding mechanism to produce the corresponding displacement. As the I/V conversion is a proportional component which is easy to calculate and can not consider it, the electric actuator can be simplified as a closed loop control system, which is composed of two parts, the relay link and the transfer function. As shown in figure 2, the input of the closed-loop control system is the voltage signal Us (the signal thought I/V conversion) and the output is the position feedback signal $\mathrm{U}_{\mathrm{f}}$. The transfer function is composed of servo motor, deceleration device and position feedback device. As can be seen from Figure 2, as long as the $|\mathrm{e}|>\mathrm{h}$, the supply voltage of the servo motor is Ua. 


\section{Determine the form of the transfer function}

After linearization, the mechanical properties of two - phase servo motor can be expressed as:

$$
M_{m}=-C_{\omega} \omega+M_{S}[2]
$$

In the formula: $M_{m}$ is the output torque of the motor, $N \cdot m ; \omega$ is motor angular velocity, $\mathrm{rad} / \mathrm{s} ; C_{\omega}=\frac{d M_{S}}{d \omega}$ is damping coefficient, which is the slope of the mechanical characteristic curve after linearization, $(\mathrm{N} \cdot \mathrm{m})\left(\mathrm{rad} \cdot \mathrm{s}^{-1}\right) ; M_{S}$ is locked-rotor torque, expressed in the power supply voltage when the rotor is locked rotor torque. $M_{S}$ can be expressed as: $\quad M_{S}=C_{M} u_{a}$

In the formula: $\mathrm{C}_{\mathrm{M}}$ can be obtained by the $\mathrm{M}_{\mathrm{S}}$ which is got by the blocking torque test of the power

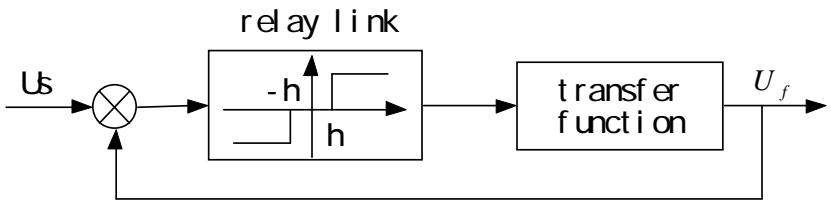

supply voltage of $\mathrm{u}_{\mathrm{a} .}$. That is $C_{M}=\frac{M_{S}}{u_{a}}(\mathrm{~N} \cdot \mathrm{m} / \mathrm{V})$.

Fig2 Schematic of the electric actuator
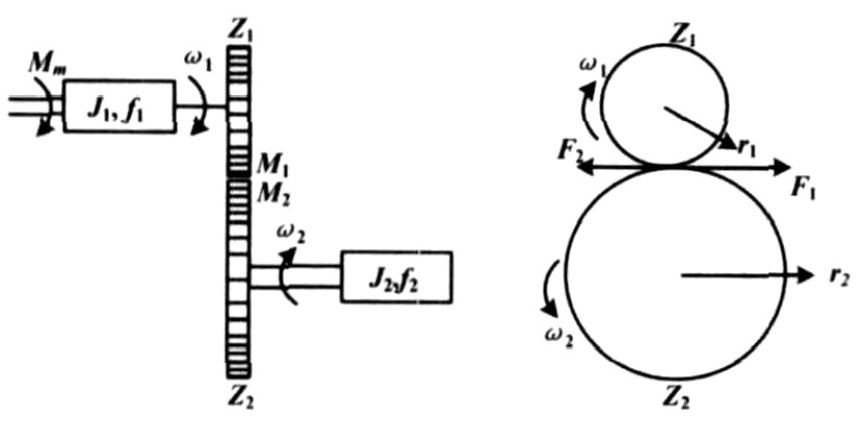

a the gear train $\quad b$ a pair of meshing gears Fig3 the gear train

The decelerator is composed of gears system and belts. Now take the gear train as an example to deduce the equation, as shown in figure 3a. In figure 3a, the angular velocity of the driving gear (gear $Z_{1}$ ) and the driven gear (gear $Z_{2}$ ) are respectively $\omega_{1}$ and $\omega_{2}$; the number of teeth of the driving gear (gear $\mathrm{Z}_{1}$ ) and the driven gear (gear $\mathrm{Z}_{2}$ ) are respectively $\mathrm{Z}_{1}$ and $\mathrm{Z}_{2}$. Obviously, the ratio of the angular velocity of gears is $\frac{\omega_{1}}{\omega_{2}}=\frac{Z_{2}}{Z_{1}}$.In the system of the servo motor and gears, the torque, the rotary inertia and the viscous friction of the load and the gear train is converted to the final gear shaft, in order to comprehend the impact that the load and the gear train influence the servo motor characteristic.

Figure $3 \mathrm{~b}$ is a pair of meshing gears, in which $\omega_{1}$ and $Z_{1}$ are the speed and the number of teeth of the gear that the radius is $R_{1}$; in which $\omega_{2}$ and $Z_{2}$ are the speed and the number of teeth of the gear that the radius is $R_{2}$. The rotation has no relative sliding, so in the meshing point of the two gears there is the same line speed that is $v_{1}=v_{2}$. At the same time, on the circumference there is equal force that is $\mathrm{F}_{1}=\mathrm{F}_{2}$.

There are $v=\omega \cdot r$ and $F=\frac{M}{r}$, so $\quad \frac{\omega_{1}}{\omega_{2}}=\frac{r_{2}}{r_{1}}=\frac{Z_{2}}{Z_{1}}[3]$

$$
\frac{M_{1}}{M_{2}}=\frac{r_{1}}{r_{2}}=\frac{Z_{1}}{Z_{2}}
$$

In the formula: $\mathrm{M}_{1}$ and $\mathrm{M}_{2}$ are respectively the resistance torque of the gear 1 and the rotational torque of the gear 2 .

For figure 3a gear, gear 1 is connected with the motor; the gear 2 is connected with the load. According to the static and dynamic method of the fixed axis rotation, there are moment balance equations of the motor axles and the load shaft: $\quad M_{m}=J_{1} \frac{d^{2} \theta_{1}}{d t^{2}}+f_{1} \frac{d \theta_{1}}{d t}+M_{1}$ 
and

$$
M_{2}=J_{2} \frac{d^{2} \theta_{2}}{d t^{2}}+f_{2} \frac{d \theta_{2}}{d t}+M_{3}
$$

In the formula: $\mathrm{M}_{\mathrm{m}}$ is the output torque of motor, $\mathrm{N} \cdot \mathrm{m} ; \mathrm{M}_{1}$ is the resistance torque $(\mathrm{N} \cdot \mathrm{m})$ that the gear 2 give the gear $1 ; \mathrm{M}_{2}$ is the angular moment of gear $2, \mathrm{~N} \cdot \mathrm{m} ; \mathrm{M}_{3}$ is the load torque of gear $2, \mathrm{~N} \cdot \mathrm{m}$; $J_{1}$ is the total inertia of motor shaft $\left(\mathrm{N} \cdot \mathrm{m} \cdot \mathrm{s}^{2}\right)$ which include the inertia of rotor and its of gear $1 ; J_{2}$ is the total inertia of the load shaft $\left(\mathrm{N} \cdot \mathrm{m} \cdot \mathrm{s}^{2}\right)$ which include the inertia of the load and its of gear $2 ; \mathrm{f}_{1}$ is the viscous friction coefficient of motor, $\mathrm{N} \cdot \mathrm{m} / \mathrm{rad} / \mathrm{s} ; \mathrm{f}_{2}$ is the viscous friction coefficient of load, $\mathrm{N} \cdot \mathrm{m} / \mathrm{rad} / \mathrm{s}$; $\theta_{1}$ is the angular displacement of the motor shaft, rad, so there is $\frac{d \theta_{1}}{d t}=\omega_{1} ; \theta_{2}$ is the angular displacement of the load shaft, $\mathrm{rad}$, so there is $\frac{d \theta_{2}}{d t}=\omega_{2}$.

Considering the formula(3), (4) and formula (6) into formula (5), that lead us to the conclusion:

$$
M_{m}=\left(J_{1} \frac{Z_{2}}{Z_{1}}+J_{2} \frac{Z_{1}}{Z_{2}}\right) \frac{d^{2} \theta_{2}}{d t^{2}}+\left(f_{1} \frac{Z_{2}}{Z_{1}}+f_{2} \frac{Z_{1}}{Z_{2}}\right) \frac{d \theta_{2}}{d t}+\frac{Z_{1}}{Z_{2}} M_{3}=J \frac{d^{2} \theta_{2}}{d t^{2}}+f \frac{d \theta_{2}}{d t}+g
$$

In the formula: $\mathrm{J}$ is the total equivalent rotary inertia; $\mathrm{f}$ is the total equivalent coefficient of the viscous friction; $g$ is a constant $\left(g=\frac{Z_{1}}{Z_{2}} M_{3}\right)$.

For the multistage gear train, step by step calculated and can get the same form with formula (7).

In the position feedback loop, there are two transformations. The first is that the angular displacement of the gear 2 (in the figure 3) is changed into the linear displacement; Second is that the linear displacement is changed into an appropriate electrical signal. The two kinds of transformation are linear transformation and the transformations can be show with $x=r \theta_{2}$ or $u_{f}^{\prime}=k \llbracket x+b$, where $\mathrm{x}$ is a linear displacement of the final stage gear; where $r$ is gear radius; where $u_{f}^{\prime}$ is electrical signals (position feedback signal which is voltage signal); where $\mathrm{k}$ and $\mathrm{b}$ are two constants. So there is a formula that is $u_{f}^{\prime}=p r \theta_{2}+b$.

According to figure1, can be known position feedback: $u_{f}=k \cdot u_{f}^{\prime}=k_{p} \cdot r \cdot \theta_{2}+k_{b}$, so there are

$$
\frac{d \theta_{2}}{d t}=\frac{1}{k_{p} r} \times \frac{d u_{f}}{d t}
$$

and

$$
\frac{d^{2} \theta_{2}}{d t^{2}}=\frac{1}{k_{p} r} \times \frac{d^{2} u_{f}}{d t^{2}}
$$

Formula (8) and (9) into (7): $M_{m}=J_{0} \frac{d^{2} u_{f}}{d t^{2}}+f_{0} \frac{d u_{f}}{d t}+g$

In the formula, $J_{0}$ and $f_{0}$ are two constants $\left(J_{0}=\frac{J}{k_{P} r}, f_{0}=\frac{f}{k_{P} r}\right)$.

Formula (1) and (2) into (10): $J_{0} \frac{d^{2} u_{f}}{d t^{2}}+f_{0} \frac{d u_{f}}{d t}+g=-C_{\omega} \omega+C_{M} u_{a}$

In the formula, $\omega$ is the angular speed of motor, from the formula (1) and (2), can draw a conclusion: $\omega=\omega_{1}=\frac{Z_{2}}{Z_{1}} \omega_{2}=\frac{Z_{2}}{Z_{1}} \cdot \frac{d \theta_{2}}{d t}=\frac{Z_{2}}{Z_{1} k_{P} r} \cdot \frac{d u_{f}}{d t}$.

So formula (11) can be changed: $J_{0} \frac{d^{2} u_{f}}{d t^{2}}+\left(f_{0}+\frac{C_{\omega} Z_{2}}{Z_{1} k_{P} r}\right) \cdot \frac{d u_{f}}{d t}=C_{M} u_{a}-g$.

The above formula can be simplified to $\frac{d^{2} u_{f}}{d t^{2}}+a \frac{d u_{f}}{d t}=k u_{a}$ 
In the formula: $a=\frac{f_{0}}{J_{0}}+\frac{C_{\omega} Z_{2}}{J_{0} Z_{1} k_{P} r}$ and $k=\frac{C_{M}}{J_{0}}-\frac{g}{J_{0} u_{a}} ; u_{a}$ is the power supply voltage of the motor. Assuming that the fluctuations of the supply voltage are negligible, the $\mathrm{k}$ can be regarded as a constant. By the Laplace transform from the formula (12), can obtained: $\quad \frac{U_{f}(s)}{U_{a}(s)}=\frac{k}{s(s+a)}$

Formula (12) and (13) are the time domain model and open-loop transfer function of the electric actuator. The model and the function are got when the power supply voltage is $u_{a}$; the feedback voltage $\mathrm{u}_{\mathrm{f} .}$ If the actuator is different, the value of $\mathrm{K}$ and a are also different.

\section{The parameters of open loop transfer function}

There is a second order ordinary differential equation: $\frac{d^{2} u_{f}}{d t^{2}}+a \frac{d u_{f}}{d t}=k u_{a}$ [4]; Its solution is

$$
u_{f}(t)=R t+B+\frac{1}{a^{2}} e^{-a\left(t+c_{1}\right)}
$$

Formula: $R=\frac{k u_{a}}{a} ; B=\frac{c_{2}}{a}\left(\mathrm{C}_{1}\right.$ and $\mathrm{C}_{2}$ is the constant which need determined $)$.

From formula (14), we can know that $\frac{1}{a^{2}} e^{-a\left(t+c_{1}\right)}$ is a very small value when time $t$ is long. The value can be ignored because of being small. $\mathrm{u}_{\mathrm{f}}$ is similar to a straight line when time $\mathrm{t}$ is long. We can calculate $\mathrm{R}$ and $\mathrm{B}$ if we take two points on the P1、P2 straight lines of $\mathrm{u}_{\mathrm{f}}$.

By differentiating formula (14), can derive the formula: $\frac{d u_{f}}{d t}=R-\frac{1}{a} e^{-a\left(t-c_{1}\right)}$. When $\mathrm{t}=0$, the motor angular velocity(the derivative of angular displacement) is $\omega=\omega_{1}=\frac{d \theta_{1}}{d t}=0$. There are $\frac{d u_{f}}{d t}=R-\frac{1}{a} e^{-a c_{1}}=0$ and $u_{0}=B+\frac{1}{a^{2}} e^{-a c_{1}}$ when $\mathrm{t}=0, \mathrm{u}_{0=} \mathrm{u}_{\mathrm{f}}$.

To sum up, can derive: $R=\frac{1}{a^{2}} e^{-a c_{1}}, u_{0}=B+\frac{1}{a^{2}} e^{-a c_{1}}, R=\frac{u_{a} k}{a}$ and $B=\frac{c_{2}}{a}$. From this four formulas, can derive: $a=\frac{R}{u_{0}-B}$ and $k=\frac{R^{2}}{\left(u_{0}-B\right) u_{a}}$.

\section{conclusion}

By the above method, the transfer function of the actuator can be calculated quickly, if we get the power supply voltage $\left(\mathrm{u}_{\mathrm{a}}\right)$ of the servo motor, the initial value $\left(\mathrm{u}_{0}\right)$ of feedback voltage $\left(\mathrm{u}_{\mathrm{f}}\right)$, two feedback voltage when servo motor running time is longer. This method is easy and quick to derive the transfer function of the actuator, because the parameter of the voltage and the time are easy to obtain.

\section{References:}

[1] Dong Yan. Research of Variable Frequency Speed System for Intelligent Electric Actuator [J]. process automation instrumentation, 2010 (9): 9-12.

[2] Hu Shousong. The principle of automatic control (1) [M]. Beijing: National Defense Industry Press, 1991:32-37.

[3] Zou Zhihong. Theoretical mechanics (Second Edition) [M]. Beijing: tsinghua university press, 2014:122-125.

[4] Teaching and research section of Tongji University. Higher mathematics. (2) [M]. seventh edition. Beijing: Higher Education Press, 2014:360-366. 